\title{
The misdiagnosis severity score and doctors' perception of the severity of diagnostic errors
}

\author{
H R Guly
}

\begin{abstract}
Objective-To test the previously described misdiagnosis severity score (MSS) by comparing it with doctors' perceptions of the severity of diagnostic errors.

Methods-14 scenarios of diagnostic errors were described on a questionnaire. These were given to 12 accident and emergency $(A \& E)$ consultants who were asked to indicate their assessment of the severity of the errors on a scale of 1 to 10 .

Results-There was a highly significant concordance between doctors' rankings of the diagnostic errors, and between the MSS and the mean severity as assessed by the A\&E consultants.

Conclusions-It is reasonable to use the MSS as a measure of the severity of diagnostic errors.

(F Accid Emerg Med 1997;14:293-294)
\end{abstract}

Keywords: accident and emergency department; misdiagnosis; severity scoring

Effective quality control in an accident and emergency department requires that diagnostic errors be audited and that their severity be described. The misdiagnosis severity score (MSS) is a scale which indicates the severity of diagnostic errors on a scale of 1 to 7 . It is obtained by adding two scores which indicate the additional treatment which a patient would have received and the follow up which would have been organised if the correct diagnosis had been made initially. It has been described elsewhere. ${ }^{1}$ The MSS has been found to be useful in the description of the diagnostic errors made in one $A \& E$ department. ${ }^{1}$

However, any such scale should be validated and must seem reasonable to those who use it. Validation needs to be done against an objective measure of outcome, but the consequences of a diagnostic error are difficult to measure. There may be no adverse consequences if the missed injury is minor, if the diagnosis was corrected quickly, or if the patient had correct treatment, despite an incorrect diagnosis. More severe diagnostic

\section{Methods}

Fourteen scenarios of injuries being misdiagnosed were circulated to $12 \mathrm{~A} \& \mathrm{E}$ consultants in the then South West and Wessex regions. These are summarised in table 1. Each consultant was asked to grade the severity of the diagnostic error on a scale of 1 to 10 . They were told that they might wish to consider whether treatment would have been different had the injury been correctly diagnosed. They were asked to grade the anatomical severity of the injury and the potential for pain, suffering, morbidity and mortality, and the risk of complaint and legal action resulting from the misdiagnosis. The MSS of each of these misdiagnoses was also calculated and compared with the median severity as assessed by the consultants.

\section{Results}

The doctors' assessments of the severity of the misdiagnoses are shown in table 2 . (In patient 14 , one doctor scored the severity as 1 but said that if the same error had been made by a surgical registrar, he would have rated the severity as 9 . Both sets of figures are shown in the table but the statistics are calculated using the score of 1.)

The concordance between doctors assessments of severity was tested using the Kendall coefficient of concordance. This was highly significant $\left(\mathrm{W}=1.23, \chi^{2}=125.5, \mathrm{P}<0.001\right)$.

This highly significant concordance allowed testing of the correlation between the median severity as estimated by the consultants and the MSS. This is shown in fig 1 . The correlation is highly significant (Spearman rank coefficient $r_{s}=0.902, P \ll 0.001$.)

\section{Discussion}

When asked to judge the severity of a diagnostic error on a scale of 1 to 10 , there was wide

\section{Table 1 Scenarios for validation}

\begin{tabular}{ll}
\hline Patient & Diagnosis \\
\hline 1 & Haemarthrosis of elbow (positive fat pad sign) \\
2 & Epiphyseal fracture of distal radius \\
3 & Child with spiral fracture of tibia and intact fibula \\
4 & Fracture of tuft of terminal phalanx of finger \\
5 & Ruptured Achilles tendon \\
6 & Severe crush fracture of calcaneum \\
7 & Fracture of 8th rib \\
8 & Small pneumothorax \\
9 & Central cervical cord contusion \\
10 & Laceration flexor digitorum profundus tendon \\
11 & Fracture of base of 3rd metatarsal \\
12 & Fractured neck of femur \\
13 & Fractured medial malleollus in a patient admitted \\
& with a head injury \\
14 & Jejunal perforation in a patient with lack of sensation \\
& due to tetraplegia \\
\hline
\end{tabular}
\footnotetext{
errors may cause pain and increased length of stay in hospital and may risk disability or even death. Even minor errors may lead to complaint or poor publicity and more serious errors may lead to legal action, though this may not be initiated for several years. There is no one outcome measure against which the severity of all diagnostic errors can be validated and so the MSS has been tested against senior doctors' perception of error severity. Dr H R Guly, consultant in

\section{Accepted for publication 10 April 1997 \\ Accident and
Emergency \\ Department, H R Guly \\ Correspondence to: $\mathrm{A} \& \mathrm{E}$ Plymouth PL6 8DH}


Table 2 Severity of misdiagnosis as indicated by 12 consultants

\begin{tabular}{llll}
\hline \multirow{2}{*}{ Patient } & \multicolumn{3}{l}{ Severity of injury } \\
\cline { 2 - 4 } & Range & Median & Interquartile range \\
\hline 1 & $2-4$ & 3 & $2-3$ \\
2 & $2-8$ & 3.5 & $2.25-6.5$ \\
3 & $3-8$ & 6 & $5-7$ \\
4 & $1-5$ & 2 & $1-3.75$ \\
5 & $4-8$ & 5.5 & $5-7$ \\
6 & $4-9$ & 7.5 & $6.25-8$ \\
7 & $1-4$ & 2 & $1-3$ \\
8 & $3-8$ & 4.5 & $3.25-5.75$ \\
9 & $2-9$ & 5 & $4-7.75$ \\
10 & $3-9$ & 6.5 & $5.25-8.75$ \\
11 & $1-5$ & 2 & $1.25-2.75$ \\
12 & $2-8$ & 6.5 & $4.25-7$ \\
13 & $2-6$ & 3 & $2.25-4$ \\
14 & $1-10$ & 8 & $3.75-8.75$ \\
& $(3-10)$ & $(8)$ & $(6.25-9)$ \\
\hline
\end{tabular}

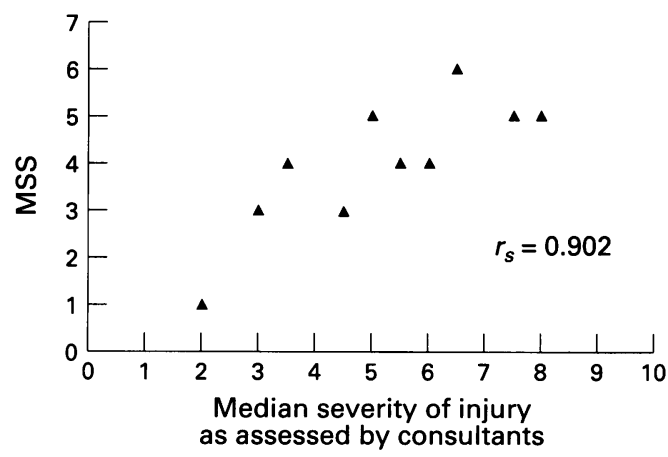

Figure 1 Misdiagnosis severity score (MSS) of the 14 misdiagnoses plotted against the median severity of injury as assessed by 12 consultants (some points on the graph represent two patients).

variation between individual doctors, for example, the seriousness of missing a fractured neck of femur was scored from 2 to 9 . Despite this, the concordance between doctors' rankings of the severities of missed injuries is highly significant.
There are theoretical difficulties in calculating the MSS by the addition of two non-linear scores. However, the important factor is whether the MSS (however derived) correlates with any other measure of severity. The significant concordance between doctors' assessment of severity allows comparison of the median of their scores with the 7-point MSS, and the correlation was highly significant. The MSS compares well with experienced doctors' assessment of severity and can reasonably be used as a measure of the severity of diagnostic errors.

The consultant who gave different scores to patient 14 depending on the experience of the doctor making the error was clearly basing the assessment of severity on the degree of negligence by an individual. However, the MSS is a measure of the potential seriousness of a diagnostic error to the patient and to the hospital. An inexperienced doctor making a serious error may not be personally negligent but the patient is at no less risk of harm because the error has been made by a junior doctor, and the hospital may be at medicolegal risk for poor supervision of the doctor, poor protocols, etc. The MSS should not be a measure of individual blame.

The wide variability of doctors' assessment of the severity of individual injuries is more reason for an easily calculated severity scale to replace subjective judgement in indicating the severity of a diagnostic error for the purposes of audit. The MSS can be used for this purpose. It should not be used as an indicator of severity for medicolegal purposes or for indicating the consequences of an error for an individual patient.

I would like to thank Dr C Ricketts, Department of Mathematics and Statistics, University of Plymouth, for statistical advice.

1 Guly HR. A scale for measuring the severity of diagnostic errors in accident and emergency departments. J Accid Emerg Med 1997;14:290-2. 